\title{
Yellow Fever: A Re-Emerging Threat
}

\section{Yunfei Zhao ${ }^{1 *}$, Xiyun Zhang², Siyi Shu ${ }^{3}$, Yijia Sun ${ }^{4}$, Xiangxiong Feng5 ${ }^{5}$, Shizhong Zhang ${ }^{6}$}

${ }^{1}$ The World Leading Schools Association (Fudan Academy), Shanghai, China

${ }^{2}$ Suzhou Middle School, Jiangsu, China

${ }^{3}$ Jia Xiang Foreign Language School, Chengdu Seventh Middle School, Chengdu, China

${ }^{4}$ Harbin Shun Mai Foreign Language School, Harbin, China

${ }^{5}$ Zhengzhou Ninth Middle School, Zhengzhou, China

${ }^{6}$ Beijing Royal School, Beijing, China

Email: *yunfeizhao@yahoo.com

How to cite this paper: Zhao, Y.F., Zhang, X.Y., Shu, S.Y., Sun, Y.J., Feng, X.X. and Zhang, S.Z. (2018) Yellow Fever: A Re-Emerging Threat. Health, 10, 1431-1448. https://doi.org/10.4236/health.2018.1010110

Received: September 26, 2018

Accepted: October 28, 2018

Published: October 31, 2018

Copyright $\odot 2018$ by authors and Scientific Research Publishing Inc. This work is licensed under the Creative Commons Attribution International License (CC BY 4.0).

http://creativecommons.org/licenses/by/4.0/

\section{cc) (i) Open Access}

\begin{abstract}
Yellow fever (YF) is arguably one of the most notorious infectious diseases in the world. The disease is not only fatal to the human but also several primate species many of which are endangered by now. YF is caused by the yellow fever virus (YFV). While YFV was one of the most feared infectious diseases in the 18th and 19th century, the overall disease burden has been greatly reduced through an effective vector (mosquito) control and the development of the live-attenuated vaccine, YFV-17D. However, recent outbreaks in previously non-endemic areas have risen intense awareness among scientists and the public and remind us that YFV cannot be forgotten and containing it needs to remain a global health priority. Notably, the 11 imported yellow fever cases to China; hence the whole Asia-Pacific region, in 2016 alone, highlight that YFV may pose a threat to a large population which is intensified by increasing human migration and an extremely low vaccination rate. This paper assesses the possibility of a new round of epidemics of YFV, indicating that the virus should indeed be recognized as a re-emerging threat, and offers suggestions on how to prevent it. Stricter vaccine regulation and border check should be applied, as well as further research into alternative vaccines. More attention and efforts should be paid to fighting against the disease.
\end{abstract}

\section{Keywords}

Yellow Fever, Global Epidemics, Research Essay, Vaccination Quarantines

\section{Introduction}

\subsection{A Brief History of Yellow Fever}

Yellow fever remains as one of the most dangerous infectious diseases in the 
world. Outbreaks of yellow fever mainly occur in Africa and South America. Each year approximately 80,000 - 200,000 people become infected and approximately 60,000 succumb to the infection [1]. Yellow fever is thought to have originated in Africa and its etiologic agent-the yellow fever virus (YFV)-is transmitted by Aedes aegypti, a species of mosquito. Around 400 years ago, YFV was spread to North and South America by the slave trade and caused a series of outbreaks in many cities in the Americas [2]. During the 1980s, the number of infected people increased dramatically; probably because of more urban residents and frequent population migration [3]. Though people tried to control the spread of the main YFV vector, $A$. aegypti, and confine the virus in the jungle by some vaccination programs, the mosquito and YFV still keeps re-emerging in endemic areas and there are no effective treatments for YFV. In 2006, in order to relive such severe yellow fever situation, the World Health Organization (WHO) sponsored routine immunization of children in Africa and adopted the vaccination. However, the vaccination rates still remain low [4].

\subsection{Molecular Virology}

YFV belongs to the Flaviviridae, more specifically as a classical Flavivirus, the same genus which other infectious viruses like dengue virus and West Nile virus belong to. YFV is an enveloped virus with a positive-sense, single strained RNA molecule composed of about 11,000 nucleotides inside encoding a single open reading frame (ORF). Mature virions are spherical particles that are approximately $50 \mathrm{~nm}$ in diameter. The lipid envelops are derived from host cell membrane, along with the assistance of the three structural proteins (C(Capsid)$\operatorname{PrM}($ Membrane Precursor)-E(Envelop)) encoded by the N-terminal quarter of the virus genome [5] [6]. The rest of the coding region codes for the seven nonstructural (NS) proteins (NS1-NS2A-NS2B-NS3-NS4A-NS4B-NS5) that are critical for viral functions such as RNA replication and polyprotein processing [7] [8]. Processing of the YFV polyprotein is facilitated by a virus-encoded serine protease (NS2B/3) as well as host signal peptidase [6]. Other host proteins, like the Hsp40 chaperone protein (DNAJC14), may also interact with the virus and serve as important regulation factors [9]. The remaining genome of the virus is the non-coding region (NCR). The $3^{\prime}$ NCR varies in size between YFV strains of different genotype [5]. Stem-loops formed near the 3' and 5' terminals of the $\mathrm{NCR}$ are found to be essential in virus replication [6] [10].

The life cycle of YFV, though many processes and factors have been revealed, is still not completely understood. First, the glycoproteins on the YFV surface bind to one or more yet-to-be identified receptor(s) on host cells [6] and triggers endocytosis. The E protein, which contains a fusion peptide, undergoes trimerization in the acidic environment of the vesicle and promotes fusion of the viral and host membrane to release YFV genome into the cytoplasm. Then, viral RNA is replicated and translated, and the virus is assembled on the endoplasmic reticulum (ER) with the structural proteins [11]. It was found that the proper fold- 
ing of the E protein needs the aid of PrM [12]. As the virus particles mature, the $\operatorname{PrM}$ is cleaved into Pr and M by host protease furin, which leads to the rearrangement of the E protein [11] [13]. The virions are then transported to the Golgi apparatus and are ready to be released.

\subsection{Transmission}

Yellow fever is an acute infection caused by YFV and transmitted by mosquitoes [14] [15]. The disease is endemic mainly in tropical regions of central and South America and Africa, with periodic natural infection cycles between mosquitoes and non-human primates. Yellow fever is a zoonotic disease of humans and animals [16]. YFV is also circulating in wild monkey species, e.g. in the tropical jungles of Africa and South America, which are bitten by $A$. aegypti and then transmitted to other monkeys. When humans enter these jungles, they are likely to become infected. This is a common form known as the sylvatic cycle. People who have been infected with YFV can also be a source of infection, as they transmit the virus to others through the bite of $A$. aegypti that occupy urban areas. A. aegypti mosquitoes can survive on airplanes and ships as well [17]. This is known as the urban cycle.

\subsection{Prevalence}

Since the turn of the 20th century, yellow fever has been endemic mainly in tropical regions of Africa and Latin America (i.e., Mexico and South America), where more than 900 million people are threatened by yellow fever. The WHO statistics estimate that 200,000 people are infected every year, with 30,000 deaths and 90 percent of which are in Africa. According to data from WHO, yellow fever is endemic in 47 countries, including 34 in Africa and 13 in central and South America (Figure 1 and Figure 2). In 2015, the WHO recognized the risk of yellow fever epidemics in 39 countries or regions, and requested travelers to be vaccinated against yellow fever in those countries [15].

On 26 January 2016, Angola reported an outbreak of yellow fever through IHR, according to a WHO report dated February 12th, 2016. The first case was detected on 5 December 2015 in the region of Viana, Luanda. A total of 2148 suspected yellow fever cases have been reported in Angola, including 641 laboratory confirmed cases and 277 deaths. The WHO called the outbreak a "serious public health event". Other affected areas include Cabinda, South Kwanza, Huambo, Ulaya and Uige [15] (Figure 3).

YFV is not endemic to (South) East Asia in general and China specifically and the current cases of yellow fever have all been imported. The patients entered the country after becoming ill in the endemic areas. Yellow fever imported cases have been found in Beijing, Fujian and Shanghai [18].

\subsection{Symptoms, Diagnosis, and Available Treatment of YFV}

Even though YFV could result in severe physiological responses, the disease 

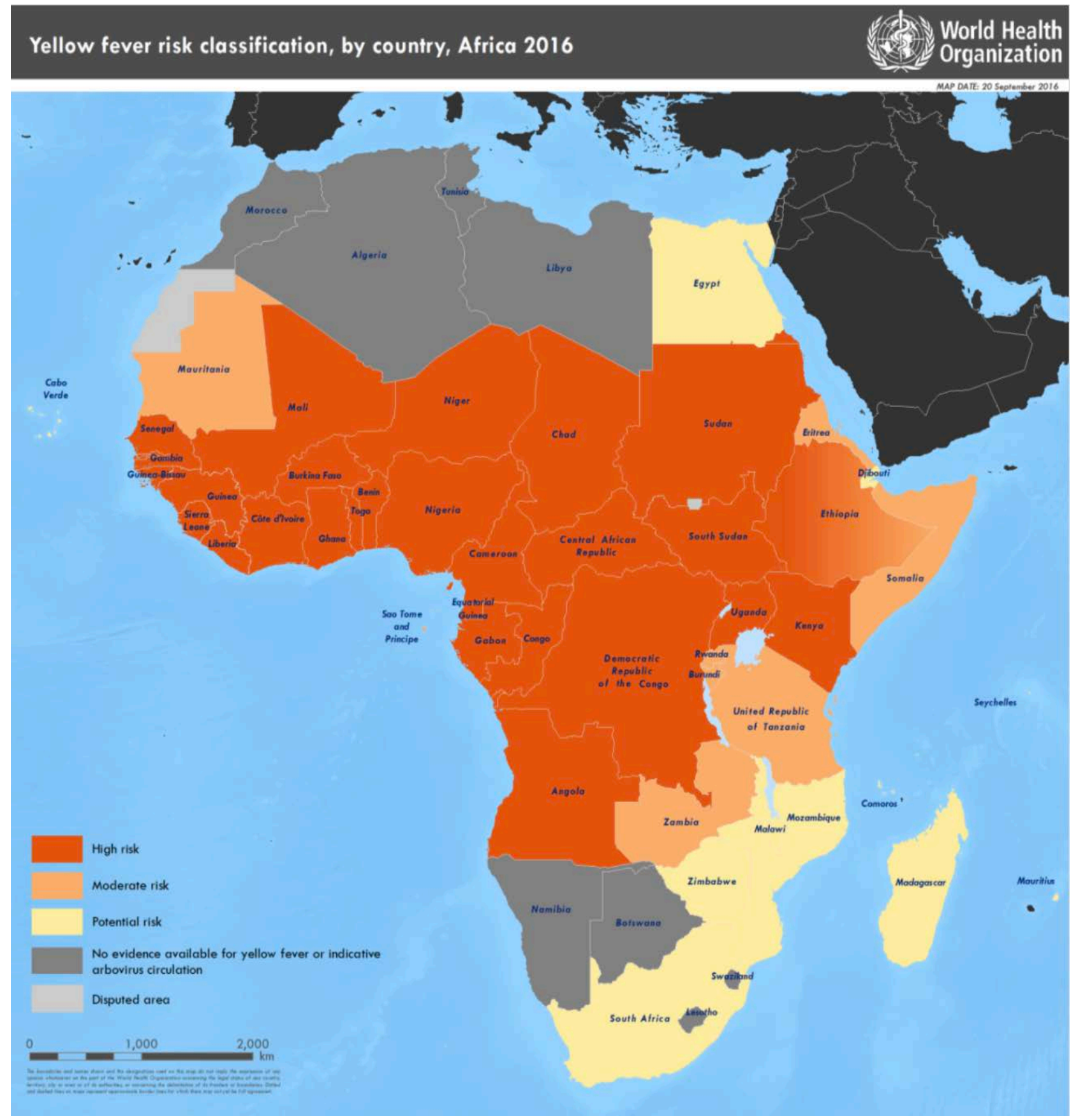

Figure 1. Risk of YF outbreaks in Africa by country, 2016 [63].

symptoms are not immediately observable until an incubation period has passed. The incubation period between an infection and initial symptoms is usually three to six days, then following with one or two phases of illness [19]. Most people infected with YFV first go through an acute phase with relatively mild symptoms, including fever, headache, chills, back pain, fatigue, loss of appetite, muscle pain, nausea, and vomiting, which lasts for roughly three to four days. Majority of infected people usually recovers after the brief acute phase, yet still $15 \%$ could enter a second, toxic phase, which is characterized by much more 


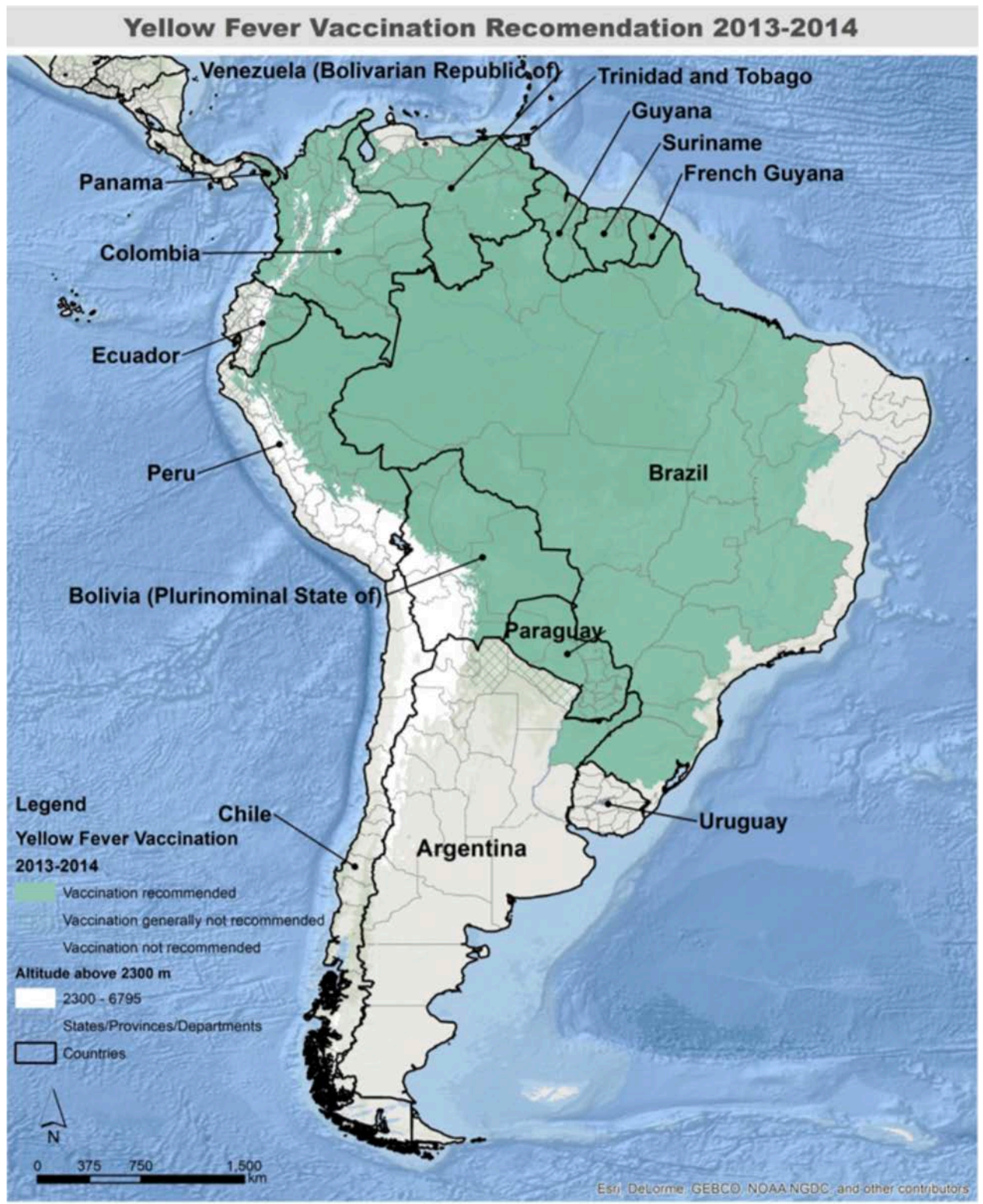

The boundaries and names shown and the designatons used on this map do not impy the expression of any opinion whatsor on the part of the Wond Heath Organization concerning the legal statis of any country, teritory, cily or area or of is authorit or concerning the delimtation of its fronters or boundaries. Doted and dashed ines on maps represent apgroximate border for which there may not yet be $\mathrm{H}$ agreement.

Data Sources: World Heath Organization. Pan American Heath Organization. Member States

\section{Pan American Health Organization} Map Production: PAHO-WHO AD CHA IHR. Epidemic Alert and Response, and Water Bome Diseases

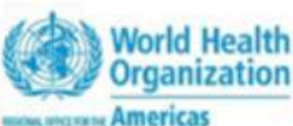

Figure 2. YF risk areas in South America, Panama, and Trinidad and Tobago [64]. 


\begin{tabular}{|c|c|c|c|c|c|c|c|}
\hline & Country & \begin{tabular}{|l|} 
Numbers of YF \\
outbreaks 1990- \\
2016 \\
\end{tabular} & \begin{tabular}{|l} 
Recent \\
report of YF \\
cases $^{1}$
\end{tabular} & $\begin{array}{l}\text { National PMVC } \\
\text { prior to the YFI }\end{array}$ & High seroprevalence $^{2}$ & $R o \geq 1.25$ & Risk level \\
\hline 1 & Angola & 1 & $Y$ & & & & High \\
\hline 2 & Benin & & & $Y$ & & $Y$ & High \\
\hline 3 & Burkina Faso & 5 & & $Y$ & & $\mathrm{Y}$ & High \\
\hline 4 & Cameroon & 5 & $Y$ & $\mathrm{Y}$ & & & High \\
\hline 5 & C. A. R. & 3 & & & & $Y$ & High \\
\hline 6 & Chad & 1 & $Y$ & $Y$ & & & High \\
\hline 7 & Congo & 2 & $Y$ & & & $\mathrm{Y}$ & High \\
\hline 8 & Côte d'Ivoire & 7 & $Y$ & $Y$ & & $\mathrm{Y}$ & High \\
\hline 9 & DRC & 4 & Y & & $Y$ & & High \\
\hline 10 & Eq. Guinea & & Y & & & & High \\
\hline 11 & Ethiopia & 1 & Y & & $\mathrm{N}$ & & High \\
\hline 12 & Gabon & & $Y^{3}$ & $Y$ & & $\mathrm{Y}$ & High \\
\hline 13 & Gambia & & & $Y$ & & $Y$ & High \\
\hline 14 & Ghana & 1 & $Y$ & & & $Y$ & High \\
\hline 15 & Guinea & 10 & & $Y$ & & $Y$ & High \\
\hline 16 & Guinea-Bissau & & $Y^{4}$ & & & $\mathrm{Y}$ & High \\
\hline 17 & Kenya & 2 & & & $\mathrm{~N}$ & & High \\
\hline 18 & Liberia & 5 & & Y & & $\mathrm{Y}$ & High \\
\hline 19 & Mali & 2 & & & & $\mathrm{Y}$ & High \\
\hline 20 & Niger & & & & & $Y$ & High \\
\hline 21 & Nigeria & 3 & & & & $Y$ & High \\
\hline 22 & Senegal & 5 & $Y$ & $Y$ & & $Y$ & High \\
\hline 23 & Sudan & 4 & $Y$ & & $Y^{5}$ & & High \\
\hline 24 & South Sudan & 1 & & & $Y^{6}$ & & High \\
\hline 25 & Sierra Leone & 3 & & & & $\mathrm{Y}$ & High \\
\hline 26 & Togo & & & $Y$ & & $\mathrm{Y}$ & High \\
\hline 27 & Uganda & 2 & $Y$ & & $Y$ & & High \\
\hline 1 & Burundi & & & & & $N$ & Moderate \\
\hline 2 & Eritrea & & & & & $N$ & Moderate \\
\hline 3 & Mauritania & & & & & $\mathrm{N}$ & Moderate \\
\hline 4 & Rwanda & & & & $\mathrm{N}$ & $\mathrm{N}$ & Moderate \\
\hline 5 & Sao Tome and P. & & & & & NA & Moderate \\
\hline 6 & Somalia & & & & & $\mathrm{N}$ & Moderate \\
\hline 7 & $\begin{array}{l}\text { Tanzania (United } \\
\text { Republic of) }\end{array}$ & & & & & $N$ & Moderate \\
\hline 8 & Zambia & & & & $\mathrm{N}$ & $\mathrm{N}$ & Moderate \\
\hline
\end{tabular}

Ro = basic reproductive number; PMVC = preventive mass vaccination campaign; $\mathrm{YFI}=$ Yellow fever Initiative; $\mathrm{Y}$ = yes; $\mathrm{N}=$ no; $\mathrm{NA}=$ not available.

- In Ethiopia, the YF risk assessment found evidence of risk and virus circulation limited to the south-western part of the country. South-western Ethiopia only is therefore considered to be at high risk.

- PMVCs conducted in the 1940s-1960s, except for Gabon and the Gambia, which conducted national mass campaigns in response to epidemics in 1995 and 1979, respectively.

Figure 3. Risks of YF virus circulation of 35 African countries [61].

severe pain and damage to human body. Such illness could be observed through decreased urination, abdominal pain, vomiting (sometimes with blood), heart rhythm problems, seizures, delirium, and bleeding from mouth, nose, and eyes. Infectors who enter this phase would face a fatality rate of $50 \%$ to above [20].

The diagnosis of yellow fever is usually based on the travel history and laboratory testing besides the physical symptoms displayed. Especially because of the similarity between yellow fever's initial symptoms and those of other influenza 
viruses, knowing the travelling history related to epidemic areas is very essential [21]. Despite the clinical diagnosis, YFV could be more precisely detected by reverse transcription polymerase chain reaction (RT-PCR), which amplifies short conserved segments of the YFV genome. Yet this specific method is only feasible under laboratory circumstances, as such direct operations on patient could cause further disease and is impractical.

There is currently no medical cure for yellow fever, whereas the possible treatments are basically focusing on ameliorating disease symptoms, including resting, applying fluids, and using pain relievers. Certain medications should be avoided, such as aspirin or other nonsteroidal anti-inflammatory drugs, which may increase the risk of bleeding. Severely infected patients should be hospitalized for supportive observations and prevention from further spread of the virus, especially in a non-epidemic area [22].

\subsection{Vaccination}

Although the lack of viable treatment for the yellow fever makes the disease more life-threatening, there is currently a live-attenuated $17 \mathrm{D}$ vaccine for yellow fever the WHO recommends as a precaution, including two substrains 17D-204 and 17DD [23]. The yellow fever vaccine is demonstrated to be safe with more than 500 million doses given and extremely few cases of vaccine-induced illnesses. Possible mild side effects resulting from the vaccine include headache, muscle pains, pain at the injection site, fever, and rash [24]. The frequencies of adverse events following immunization (AEFI), when the vaccine strain spreads to infection of the liver, the kidneys or the nervous system, are as low as 0 to 0.21 cases every 10,000 doses in yellow-fever-endemic regions, and from 0.09 to 0.4 cases per 10,000 doses in populations who are not exposed to the virus constantly [25]. More specifically, there would be cases of severe allergies in approximately eight per million doses, severe neurological problems in roughly four per million doses, and grave organ failure problems in three per million doses [24]. In addition, the YFV vaccine is proven to be one of the most effective vaccines ever created. Once vaccinated, people are most likely to develop immunity within ten days and then safeguarded from the virus in more than $99 \%$ of the cases [24]. The protection is lifelong, without the need of a booster ten years after the first dose [26]. There is also currently no record of the vaccine particularly disfavoring certain ethnic groups. With high efficacy and low side effects rate, the yellow fever vaccine is listed on the WHO's List of Essential Medicines, demonstrating its own reliability [27]. Thus, it is recommended that people from age nine months or older and who plan to travel to or currently reside in risky areas for yellow fever virus in Africa and South America receive vaccination [28]. Wide-spread distribution of the vaccine is crucial to the inhibition of the outbreak, and ultimately beneficial by building up herd immunity, the specific threshold of the proportion of immune individuals in a given population that will lead to the decline or elimination of the incidence of the disease [29]. Ac- 
cording to WHO, a minimum of $80 \%$ of vaccine coverage in a certain population is required to prevent further outbreaks and possibly reach the threshold for herd immunity [30]. Studies have shown that current vaccine coverage differs greatly from one risk zone to another, ranging from $100 \%$ in parts of Brazil, to $0 \%$ in parts of Central and East Africa [31]. Coverage was recently increased in West and Central Africa due to large preventive campaigns, thanks to the support of the Yellow Fever Initiative. Regardless, reports still indicate an overall low coverage in most of the risk zones, especially large areas of central and east Africa and parts of Nigeria along with other countries. In South America, low coverage was also noted [31]. With such data, researchers are able to calculate the number of people susceptible to the disease and in need of vaccination to reach herd immunity. Moreover, it was shown that introducing or communicating the concept of herd immunity improved willingness to vaccinate, therefore it would be reasonable to conclude that the prevention measure will be best carried out by large preventive campaigns on vaccination, especially for newborns, and the promotion of herd immunity [32].

\subsection{Summary}

In this paper, we look into the impact of climate change and human migration on YFV transmission, YFV's influence on endangered primate species, as well as the safety and regulation of the current vaccines and quarantine policies. The goal of this paper is to review and evaluate these aspects to demonstrate the potential of a re-emerging YFV threat and feasible means of prevention.

By conducting researches on these relevant aspects, we conclude that there will probably be minor possibilities of deadly YF outbreak in the future. Yet the incomplete border quarantine system and vaccination regulations for travelers are ineligible hidden dangers for cross-continental spread of YFV, especially under the large-scale population migration between Africa and Asia. While the development of life attenuated 17D vaccine is providing future protection against YF epidemics, its side effects and inviability in certain groups of population remain unstable to be widely applied. Hence, further vaccine development, regulation improvements, as well as proper control of vectors and effective coverage of vaccine are necessary to eliminate the threat of YFV in the future.

\section{Research}

\subsection{YFV in Asia: A Rising Threat?}

Climate and human migration are both important contributing factors for YFV proliferation and transmission. Originating from Africa, YFV was able to colonize other parts of the world via large scale human migrations. In the 1600s, the virus was spread into America through slave trade, and had caused several catastrophic events ever since. The vector responsible for the viral transmission, $A$. aegypti, is a species of mosquito that distributes widely among tropical areas of almost all continents [4] [33]. As an ectotherm, the internal temperature of $A$. 
aegypti is linked to the ambient temperature. Therefore, its life cycle and viral replication in the mosquito carrier is largely dependent on local temperature and rainfall [34]. Some coastal areas in Asia share similar climate features with yellow fever outbreak areas. For instance, Angola and Guangdong Province in China have the same annual average temperature of around $22^{\circ} \mathrm{C}$, and roughly the same annual precipitation of around 15,000 milimeter [35]. With favorable climate conditions and relatively high vegetation coverage, many countries and regions in South Asia are considered as ideal habitats for A. aegypti, hence the possible location for yellow fever epidemics. (Figure 4) The situation can be worse in these developing Asian countries without a well-established medical and hygienic system.

It has been long disputed why China, or the whole Asia-Pacific region, has never actually suffered from this disease, despite its seemingly perfect endemic condition. Several hypotheses have been proposed to address this problem. Researchers suggested the possibility of cross-protection, which is known as the Asian hypothesis. Flavivirus, like the dengue virus and Japanese encephalitis virus, which have been endemic in Asia for a long period of time, may have induced some immunity against YFV on those who had been previously infected with these viruses [4] [36]. Also, the scale of historical slave trade between Africa and Asia was quite small and was therefore unable to induce epidemics [37]. When taken into account of the fact that East Africa strain of $A$. aegypti is a less

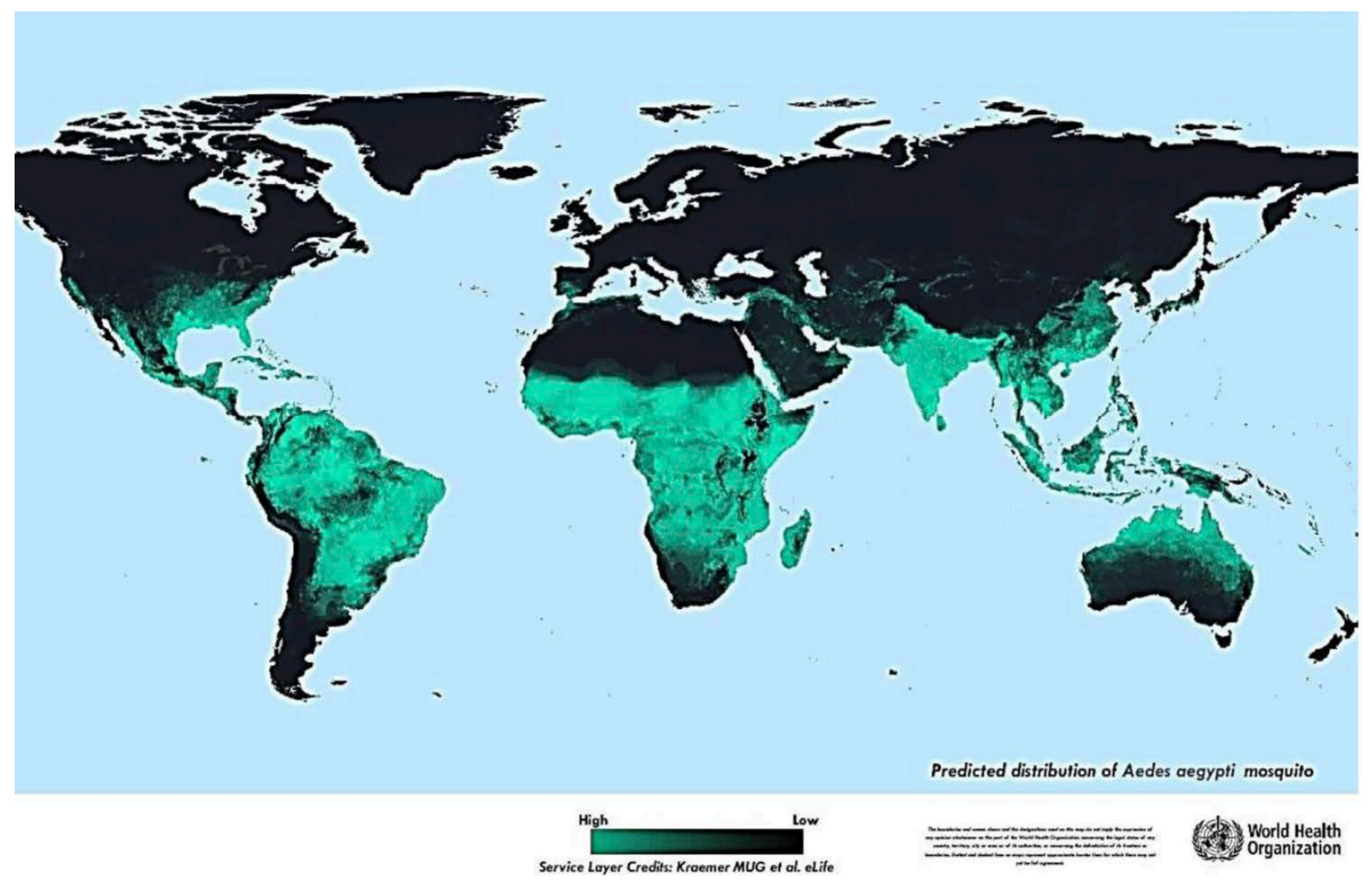

Figure 4. Probability of Occurrence of the Aedes aegypti mosquito under current environmental and land cover conditions [62]. 
favorable host for YFV and that it may be less adapted to human environment, mosquitos are thought to inhabit Asia much later than America [38].

However, as airline travels increase the flow of population between continents nowadays, the probability of viral transmission to Asia should be given more priority. As many as 45.2 million international air travelers departed from YFV endemic areas of the world in 2016, contributing to international transmission of YFV [39]. Furthermore, global warming and agricultural development may hasten the decline of sylvatic habitats for $A$. aegypti, increasing the potential contact between sylvatic and urban cycles and could directly introduce the virus into human population [40]. Our discussion is based mainly on the situation of China, which we believe would serve as an crucial implication for the future of the Asia-Pacific region.

\subsection{Yellow Fever in China}

In the March of 2016, 11 Chinese patients were diagnosed with yellow fever after returning home from their work in Angola, representing the very first cases of YFV transmission into Asia. This alarming situation revealed the possibility of imported yellow fever epidemics in Asia-Pacific region, indicating that stricter regulations should be carried out in order to ensure public health.

China has been largely invested in African countries since the mid-20th century. China and Africa have been cooperating intensively, resulting in a rapid development of tourism industry and an increasing exchange of workforce, both of which contribute greatly to the population movement. The number of Chinese travelers to African countries is increasing at a rate of $50 \%$ each year, including also travel to yellow fever endemic areas, such as Kenya [10]. Holding the biggest population within regions favoring the transmission of YFV, it is suggested that the possibility of virus transmission should not be neglected. According to a recent research based on global travelling data, China received approximately 720,000 visitors from yellow fever endemic areas in 2016 alone, it became one of the biggest populations within regions favoring the transmission of YFV [8]. Furthermore, the number of Chinese migrant workers in Africa is also ineligible. According to Johns Hopkins University SAIS China-Africa Research Initiative, the total number of Chinese labors reached 227,400 by the end of 2016, while the Algeria and Angola occupy the vast majority, holding 91,000 and 29,000 people, respectively [11]. Among these Chinese-African workers, over 105,000 of which stayed in endemic areas, as defined by WHO yellow fever risk analysis [12] (Figure 5). Suchlarge number of migrant populations yields unquestionable possibilities of the spread of YFV via international flights. The 11 cases of infected Chinese migrant workers in Africa are the direct result of human migration activities between endemic and unaffected areas; this also indicates the potential of yellow fever epidemic in Asia. The transmission of YFV in Asia needs to be eliminated through the process of vaccination and clinical treatments due to a favorable environmental condition the large quantity of suitable vectors. 


\section{Number of Chinese workers in Africa by end of year}

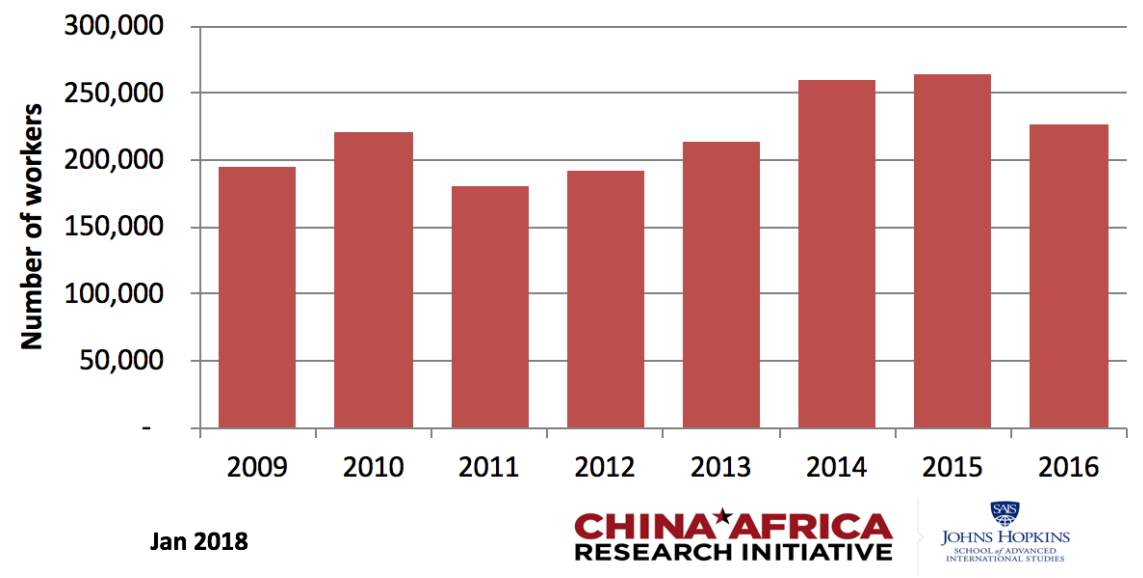

Figure 5. Number of Chinese workers in Africa by end of year 2017 [11].

The number of reported yellow fever cases could be sufficient among the total Chinese migrant workers in endemic areas with a ratio of 11 to 105,000 . While only $15 \%$ of the yellow fever cases showed clinical symptoms [41], at least 73 Chinese workers were infected [42], yielding an infection rate of $0.070 \%$. Therefore, it could be reasonably suspected that vaccination rate has not reached herd immunity among these workers. Hence, though specific data could not be obtained, the vaccination rate among Chinese migrant workers in Africa requires further enhancement. The vaccination of migrant workers should be lawfully enforced before returning from African endemic areas.

International YFV vaccination regulation states the vaccination requirement for these international migrant workers. Nevertheless, such system is not completely efficient. Invalid vaccination certificates are widely made and sold in e.g. Kenya [43], an endemic zone in Africa [44], while the validity of vaccination records in other African countries is unknown. The illegal trade and production of yellow fever vaccination certificates lead to the illegal migration departing from endemic areas, hence potential of international transmission of YFV. While surveillance on regulations is needed in Africa, Chinese custom examination reveals potential loopholes in allowing entrance from yellow fever endemic areas. According to Frontier Sanitary Quarantine Regulations of the Peoples Republic of China (PRC), travellers need to present a YFV vaccination certificate if they arrive from Yellow Fever endemic areas [45]. Despite this rule, the majority of flights departing from African countries transfer at non-endemic cities like Dubai and Johannesberg [46], whereas Chinese citizens are not required to show their boarding passes upon re-entrance, which means their original departures site are not necessarily checked. Unvaccinated Chinese migrant workers and travelers could therefore pass the border vaccination check and enter Chinese border if no clinical symptoms were exhibited.

As the largest and the most populated nation in Asia, Chinese introduction of YFV could directly affect the Asian continent, especially among the East Asian 
nations. With the existence of $A$. aegypti and the suitable environment for its prevalence, Asian nations are under substantial risks that should raise much concern on YFV prevention. The 11 cases of Chinese migrant workers have raised an alarm for the potential problems of current precaution system. With the current population of Chinese migrant workers in African endemic areas, increased vaccination rate and stricter quarantine is necessary. Corresponding companies and government institutions are responsible for the cooperating and preventing YFV transmission from Africa to Asia.

\subsection{YFV's Impact on Endangered Primate Species}

When yellow fever outbreak takes place, the local primate species, especially the Howler monkeys, are more susceptible to being infected. Among these howlers, the Brown Howlers are being infected at a higher frequency [47]. The Brown Howlers live in the Atlantic rainforests, ranging from Bahia and Espirito Santo states, Brazil, to Rio Grande do Sul and Misiones, Argentina [48].

It was estimated that there are only over 11,000 individual howler monkeys left in the world, and 10,200 of them are unprotected [49]. Howler monkeys are mostly needed to be conserved, but the number is still decreasing for that their natural habitat is being reduced and fragmented. Research indicates that howler monkeys currently present a very scattered distribution and have been severely decimated by the recent yellow fever epidemics [50].

The high YFV fatality among monkeys is a clear indicator for the spread of the disease [51]. According to a study conducted since the 1950s, the mortality rate for an infected howler monkey is much higher than that in humans with a staggering amount as high as 90\% [52]. Many outbreaks have occurred in the past, resulting in deaths of howler monkeys. In Misiones, Argentina, 59 howlers were found dead from 2007 to 2008 [47]. In 2016, the outbreak killed 80\% to $90 \%$ of the brown howler monkeys [53]. The most serious catastrophe recorded occurred in 2017 in the Atlantic rainforests of Brazil, with up to 600 deaths [54]. In the first month of 2018, 238 monkeys were found dead in Rio state, $31 \%$ of which were killed by YFV [55]. As a result, the outbreak brought attention to international organizations, governments, and even citizens all over the world.

Though vaccination has been recommended since 2017 for all Brazilians due to the previous occurrence of yellow fever outbreaks, howler monkeys are still being decimated by local communities [56]. Therefore, local actions are necessary for the conservation of the howler monkeys.

Rural inhabitants had the false belief that monkeys are the villain of YFV transmission rather than mosquitos. Instead of the massacre of monkeys, vaccination should be made readily available to them to prevent further spreading of the disease [47]. Personal efforts from the local communities are also crucial in the battle of protecting howler monkeys. For example, the efforts of a primatologist in Mexico led to the establishment of a protected area for black howler monkeys. He further devoted himself to enhancing the awareness of protecting 
monkeys [57].

Scientists have been devoting themselves to find YFV treatments not only for human patients but also for the howler monkeys. During the Brazilian outbreak, 5300 deaths of howler monkeys have been recorded. Great efforts were made by the scientists in investigating the situation and clarifying the misunderstandings of local residents [58]. If more efficient equipment and vaccines can be produced to vaccinate the monkeys, the howler monkeys may be better protected.

\subsection{Global Vaccination}

Among the preventive measures, vaccination is the most direct and effective one, as it is essential for the formation of herd immunity in a population and subsequently the prevention for future outbreaks of yellow fever. Currently, according to $\mathrm{WHO}$, there is a low vaccination plan covering the endemic African countries including Ivory Coast, Guinea, Liberia and Kenya, with rates ranging from 20\% to $60 \%$, none of which reaches the optimal coverage [29].

The low coverage rate remains an indirect cause for large scales of outbreaks in those areas, and it is crucial to provide more vaccine doses to the populations in endemic regions and enhance the regulations of immunization procedures, especially for newborns in these areas. It is illustrated that in order to reach herd immunity, a vaccine coverage rate of over $80 \%$ is required, with a security threshold of between $60 \%$ and $80 \%$ [29]. In addition, studies have shown that it would take approximately 30 years after vaccination to likely reach herd immunity and prevent large scale outbreaks. Therefore, several vaccination strategies need to be implemented simultaneously to boost the efficiency and to shorten the time expected. WHO recommends a combination of routine childhood immunization and preventive mass vaccination campaign (PMVCs), along with catch-up campaigns to maximize the population covered by vaccination.

Fortunately, due to ongoing PMVCs in regions such as Ivory Coast, Guinea, the vaccinated population immunity in endemic areas has increased notably. Nevertheless, in countries without PMVCs, including Nigeria, Kenya, the Democratic Republic of Congo, Niger and several others, YFV immunity within their respective populations remains very low [29], which indicates that on top of reaching herd immunity, other approaches should also be taken to improve the current status quo. Furthermore, by prioritizing vaccinations in high risk countries with low coverages, future outbreaks can be better contained and alleviated.

Although vaccination has been the primary concern, it is indispensable to pay attention to other prevention measures to inhibit the spread of YFV, including yellow fever disease surveillance and vector control, particularly the A. aegypti mosquitoes. The disease would be optimally controlled by several different approaches.

Meanwhile, due to marked increase of YF vaccine-associated adverse events, scientists are striving to look for alternative vaccines for YF with higher safety levels and scalability. Despite an overall low rate of incidence, severe adverse 
events resulting from yellow fever vaccination can be a variety of allergic, neurological and organ failure problems, all of which could be lethal. Recent studies have uncovered promising candidates as alternative vaccines for YFV.

A group of researchers discovered the viability of a chimera of Japanese encephalitis virus (JEV) vaccine and YFV vaccine by using the live attenuated JEV SA14-14-2 strain as a backbone and replacing the pre-membrane (prM) and envelope (E) genes with the ones from the YFV 17D vaccine [59]. It is shown that the novel chimera exhibits a lower growth rate and smaller plaque morphology, and more importantly, proves to be much less neurovirulent than the current 17D vaccine, which occasionally induces severe damage to the host. However, the nature of this chimera as a live attenuated vaccine makes it less suitable for mass production as it has to go through cell culture. Furthermore, clinical studies are required to compare the efficacy of the current 17D with the JEV/YFV chimeric vaccine.

Besides, a much safer inactivated form of YFV has recently been investigated and tested by scientists. By using a chemically inactivated YFV-17D as an antigen with an alum adjuvant, named the XRX-001 vaccine, test subjects successfully generated immune responses and produced neutralizing antibodies for the YFV [60]. The inactivated form of the vaccine would be optimal for eliminating possible side effects induced by the live attenuated vaccine. However, the scalability of this promising alternative choice needs to be further investigated.

\section{Discussion and Conclusions}

As yellow fever has been epidemic in African and South American regions, it is crucial to consider its probability of re-emergence, especially in Asia, considering the favorable climate condition and vector presence in certain Chinese provinces, as well as the large scale of migration population between Africa and Asia. The current situation of low vaccination rate, inadequate border quarantine and vaccination regulation calls for international attention on the prevention of yellow fever transmission, since already 11 cases of YF in Chinese migrant workers who returned to China from Africa have been diagnosed in 2016 alone. This more recent trends raise the concern that YFV could be spread to the Asia-Pacific region, which has been considered to be non-endemic. Stricter law enforcement on vaccination for travelers and workers is needed, whereas fake vaccination certificates and border check should be surveilled.

YFV is also infecting the primate species, e.g. howler monkeys, as people are also mistakenly viewing the infected monkeys as disease vectors rather than mosquitoes. Necessary means of quarantines need to be taken in order to prevent the spread of pathogen, and affected individuals' need accordingly. Vaccinations for endangered primates are still being developed by scientists in order to prevent further infection.

As large preventive vaccination campaigns progress, we would certainly expect fewer, and weaker YF outbreaks in the future. The live attenuated 17D vac- 
cine generally induces successful immune response in most of the vaccines and offers lifelong protection; yet we cannot ignore its reported severe side effects among an extremely small proportion of the population and the unviability of injection to those who are intolerant to certain egg proteins, etc. Thus, we are also looking forward to replacing the 17D vaccine with a more or equally effective and more significantly, safer and more accessible YF vaccine. This is the goal that we strive to accomplish in the near future. At the same time, it is imperative to understand that due to the high pathogenicity of YFV and the high mortality rate the disease can induce; it is likely not sufficient to fully control the epidemic solely by vaccination. Other prevention measures also play a crucial role in avoiding future outbreaks, including vector control and possibly the vaccination for primate species, which needs to be further investigated and verified. After all, it requires global effort to truly combat the disease and it will definitely take a long time to probably eliminate the threat of YFV to mankind. We must step by step explore ways to better deal with different scenarios across the globe and eventually overcome it.

\section{Conflicts of Interest}

The authors declare no conflicts of interest regarding the publication of this paper.

\section{References}

[1] Douam, F. and Ploss, A. (2018) Yellow Fever Virus: Knowledge Gaps Impeding the Fight against an Old Foe. Trends in Microbiology, 26, 913-928.

[2] Mir1, D., et al. (2017) Phylodynamics of Yellow Fever Virus in the Americas: New Insights into the Origin of the 2017 Brazilian Outbreak. Scientific Reports, 7, 7385.

[3] World Health Organization (2013) Yellow Fever Fact Sheet $\mathrm{N}^{\circ} 100$.

[4] Butler, D. (2016) Fears Rise over Yellow Fever's Next Move. Nature, 532, 155-156.

[5] Beasley, D.W.C., et al. (2014) Yellow Fever Virus: Genetic and Phenotypic Diversity and Implications for Detection, Prevention and Therapy. Antiviral Research, 115, 48-70.

[6] Lindenbach, B.D. and Rice, C.M. (2003) Molecular Biology of Flaviviruses. Advances in Virus Research, 59, 23-61. https://doi.org/10.1016/S0065-3527(03)59002-9

[7] Rice, C. M., et al. (1985) Nucleotide Sequence of Yellow Fever Virus: Implications for Flavivirus Gene Expression and Evolution. Science, 229, 726-733. https://doi.org/10.1126/science.4023707

[8] Bollati, M., et al. (2010) Structure and Functionality in Flavivirus NS-Proteins: Perspectives for Drug Design. Antiviral Research, 87, 125-148.

https://doi.org/10.1016/j.antiviral.2009.11.009

[9] Yi, Z., et al. (2011) Identification and Characterization of the Host Protein DNAJC14 as a Broadly Active Flavivirus Replication Modulator. PLoS Pathogens, 7, e1001255. https://doi.org/10.1371/journal.ppat.1001255

[10] Bredenbeek, P.J., et al. (2003) A Stable Full-Length Yellow Fever Virus cDNA Clone and the Role of Conserved RNA Elements in Flavivirus Replication. Journal of 
General Virology, 84, 1261-1268. https://doi.org/10.1099/vir.0.18860-0

[11] Mukhopadhyay, S., Kuhn, R.J. and Rossmann, M.G. (2005) A Structural Perspective of the Flavivirus Life Cycle. Nature Reviews Microbiology, 3, 13-22. https://doi.org/10.1038/nrmicro1067

[12] Lorenz, I.C., et al. (2002) Folding and Dimerization of Tick-Borne Encephalitis Virus Envelope Proteins prM and E in the Endoplasmic Reticulum. Journal of Virology, 76, 5480-5491. https://doi.org/10.1128/JVI.76.11.5480-5491.2002

[13] Stadler, K., et al. (1997) Proteolytic Activation of Tick-Borne Encephalitis Virus by Furin. Journal of Virology, 71, 8475-8481.

[14] WHO (1997) Dengue Hemorrhagic Fever: Diagnosis, Treatment and Control.

[15] WHO Yellow Fever. http://www.who.int/mediacentre/factsheets/fs100/en/

[16] Brady, O.J., et al. (2012) Refining the Global Spatial Limits of Dengue Virus Transmission by Evidence-Based Consensus. PLoS Neglected Tropical Diseases, 6, e1760.

[17] Barrett, A.D. and Higgs, S. (2007) Yellow Fever: A Disease That Has Yet to Be Conquered. Annual Review of Entomology, 52, 209-229. https://doi.org/10.1146/annurev.ento.52.110405.091454

[18] Jia, D., et al. (2012) Aedes-Borne Diseases Prevention and Control. Center for Disease Control and Prevention of Nanjing Command, Nanjing.

[19] CDC (2012) Yellow Fever-Symptoms and Treatment.

[20] Healthline. Yellow Fever. https://www.healthline.com/health/yellow-fever

[21] Yellow Fever. WHO. http://www.who.int/en/news-room/fact-sheets/detail/yellow-fever

[22] Huang, Y., et al. (2014) Flavivirus-Mosquito Interactions. Viruses, 6, 4703-4730.

[23] Gotuzzo, E., Yactayo, S. and Córdova, E. (2013) Efficacy and Duration of Immunity after Yellow Fever Vaccination: Systematic Review on the Need for a Booster Every 10 Years. The American Journal of Tropical Medicine and Hygiene, 89, 434-444. https://doi.org/10.4269/ajtmh.13-0264

[24] WHO (2013) Weekly Epidemiological Record. Health Section of the Secretariat of the League of Nations, 88, 269-283.

[25] World Health Organization (2008) Detection and Investigation of Serious Adverse Events Following Yellow Fever Vaccination. Geneva.

[26] World Health Organization (2014) International Travel and Health: World-Yellow Fever Vaccination Booster, June 5 2014. World Health Organization, Geneva.

[27] World Health Organization (2015) WHO Model List of Essential Medicines (19th List).

[28] https://www.cdc.gov/yellowfever/vaccine/index.html

[29] World Health Organization (2016) Global Strategy to Eliminate Yellow Fever Epidemics (EYE). Geneva.

[30] Shearer, F.M., Moyes, C.L., Pigott, D.M., et al. (2017) Global Yellow Fever Vaccination Coverage from 1970 to 2016: An Adjusted Retrospective Analysis. The Lancet Infectious Diseases, 17, 1209-1217.

[31] Betsch, C., Bohm, R., Korn, L. and Holtmann, C. (2017) On the Benefits of Explaining Herd Immunity in Vaccine Advocacy. Nature, 1, Article No. 0056.

[32] Eisen, L. and Moore, C.G. (2013) Aedes (Stegomyia) Aegypti in the Continental United States: A Vector at the Cool Margin of Its Geographic Range. Journal of Medical Entomology, 50, 467-478. https://doi.org/10.1603/ME12245 
[33] Hamlet, A., et al. (2018) The Seasonal Influence of Climate and Environment on Yellow Fever Transmission across Africa. PLoS Neglected Tropical Diseases, 12, e0006284. https://doi.org/10.1371/journal.pntd.0006284

[34] http://www.gd.gov.cn/gdgk/sqgm/zrdl/201111/t20111102_151567.htm

[35] Cathey, J.T. and Marr, J.S. (2014) Yellow Fever, Asia and the East African Slave Trade. Transactions of the Royal Society of Tropical Medicine and Hygiene, 108, 252-257. https://doi.org/10.1093/trstmh/tru043

[36] Amaku, M., Coutinho, F.A. and Massad, E. (2011) Why Dengue and Yellow Fever Coexist in Some Areas of the World and Not in Others? BioSystems, 106, 111-120. https://doi.org/10.1016/j.biosystems.2011.07.004

[37] Powell, J.R. and Tabachnick, W.J. (2013) History of Domestication and Spread of Aedes aegypti-A Review. The Memórias do Instituto Oswaldo Cruz, 108, 11-17. https://doi.org/10.1590/0074-0276130395

[38] Brent, S.E., et al. (2018) International Travel between Global Urban Centres Vulnerable to Yellow Fever Transmission. Bulletin of the World Health Organization, 96, 343-3548. https://doi.org/10.2471/BLT.17.205658

[39] Tabachnick, W.J. (2016) Climate Change and the Arboviruses: Lessons from the Evolution of the Dengue and Yellow Fever Viruses. Annual Review of Virology, 3, 2.1-2.21. https://doi.org/10.1146/annurev-virology-110615-035630

[40] World Tourism Organization. http://www.unwto.org/

[41] Johns Hopkiins University SAIS China-Africa Research Initiative (2018) Number of Chinese Workers in Africa by the End of Year.

http://www.sais-cari.org/data-chinese-workers-in-africa

[42] WHO (2018) Global Strategy to Eliminate Yellow Fever Epidemics (EYE). 25.

[43] David Control of Communicable Diseases Manual. 20th Edition, American Public Health Association, Washington DC.

[44] Siringi, S. (2002) Fake Health Certificate Racket Rife in Kenya. The Lancet Infectious Diseases, 2, 454.

[45] National Health and Family Planning Commission of PRC (2018) PRC Ministry of Health Regulations No. 2: Frontier Sanitary Quarantine Regulations of PRC. http://www.nhfpc.gov.cn/

[46] https://www.kenya-airways.com/ https://www.emirates.com https://www.ethiopianairlines.com/ https://www.flysaa.com/

[47] Wilder Smith, A. and Massad, E. (2018) Estimating the Number of Unvaccinated Chinese Workers against Yellow Fever in Africa. BMC Infectious Diseases, 18, 185. https://doi.org/10.1186/s12879-018-3084-y

[48] Holzmann, I., et al. (2010) Impact of Yellow Fever Outbreaks on Two Howler Monkey Species (Alouatta guariba clamitans and A. caraya) in Misiones, Argentina. American Journal of Primatology, 72, 475-480.

[49] Agostini, I. (2011) Brown Howler Monkeys (Alouatta guariba clamitans). https://www.speciesconservation.org/case-studies-projects/brown-howler-monkeys/ 1570

[50] Foster, B. (2018) How Many Mantled Howler Monkeys Are Left? https://animals.mom.me/many-mantled-howler-monkeys-left-3462.html

[51] Phillips, D. (2017) “We Didn’t Expect This”: A Historic Yellow Fever Outbreak Spreads in Brazil.

https://www.statnews.com/2017/04/13/yellow-fever-brazil-outbreak/ 
[52] Erikson-Davis, M. (2014) Will Yellow Fever Drive Brown Howler Monkeys to Extinction in Argentina?

https://news.mongabay.com/2014/04/will-yellow-fever-drive-brown-howler-monke ys-to-extinction-in-argentina/

[53] Barnett, A. (2017) Yellow Fever Outbreak Is Killing off Rare Monkeys in Brazil. https://www.newscientist.com/article/2119610-yellow-fever-outbreak-is-killing-offrare-monkeys-in-brazil/

[54] Boadle, A. (2017) Yellow Fever Taking Heavy Toll on Monkeys in Brazil's Rainforest. https://www.reuters.com/article/us-brazil-yellowfever-monkeys/yellow-fever-taking -heavy-toll-on-monkeys-in-brazils-rainforest-idUSKBN15F2JZ

[55] Cilfton, M. (2018) Yellow Fever Mosquitoes Now Killing at 10 Times the Rate of Pit Bulls. https://www.animals24-7.org/2018/03/25/monkey-angels-watching-over-us/

[56] Boadle, A. (2017) Yellow Fever Kills 600 Monkeys in Brazil's Atlantic Rainforest. https://www.businessinsider.com/r-yellow-fever-kills-600-monkeys-in-brazils-atlan tic-rainforest-2017-2

[57] Sailsbury, C. (2014) Local Communities Key to Saving the Critically Endangered Mexican Black Howler Monkey.

https://kidsnews.mongabay.com/2014/02/local-communities-key-to-saving-the-criti cally-endangered-mexican-black-howler-monkey/

[58] Kean, S. (2017) On the Trail of Yellow Fever.

[59] Paul, et al. (2011) Cefepime and All-Cause Mortality. Clinical Infectious Diseases, 52, 332.

[60] Yang, H., et al. (2018) Japanese Encephalitis Virus/Yellow Fever Virus Chimera Is Safe and Confers Full Protection against Yellow Fever Virus in Intracerebrally Challenged Mice. Vaccine, 36, 2450-2455.

https://doi.org/10.1016/j.vaccine.2018.03.038

[61] Monath, T.P. (2011) An Inactivated Cell-Culture Vaccine against Yellow Fever. The New England Journal of Medicine, 364, 1326-1333. https://doi.org/10.1056/NEJMoa1009303

[62] WHO (2018) Global Strategy to Eliminate Yellow Fever Epidemics (EYE). 8.

[63] WHO (2018) Global Strategy to Eliminate Yellow Fever Epidemics (EYE). 26.

[64] WHO (2018) Global Strategy to Eliminate Yellow Fever Epidemics (EYE). 30. 